\title{
Alterações do volume da via aérea superior após cirurgia bimaxilar para correção de má-oclusão esquelética Classe III: Uma série de casos
}

\author{
Upper airway volume changes after bimaxillary surgery for Class III skeletal malocclusion
}

correction: A case series

Cambios en el volumen de las vías respiratorias superiores después de la cirugía bimaxilar para la corrección de la maloclusión esquelética de Clase III: Una serie de casos

Recebido: 29/12/2021 | Revisado: 03/01/2022 | Aceito: 08/01/2022 | Publicado: 12/01/2022

Camila Lago
ORCID: https://orcid.org/0000-0003-0254-5648
E-mail: cami-lago@ @otmail.com
Pontifícia Universidade Católica do Rio Grande do Sul, Brasil
João Francisco Barbosa Cordeiro
ORCID: https://orcid.org/0000-0001-9977-6391
Universidade do Oeste de Santa Catarina, Brasil
E-mail: jfcbarbosa16@gmail.com
Leandro Eduardo Klüppel
ORCID: https://orcid.org/0000-0003-0437-7211
Universidade Federal do Paraná, Brasil
E-mail: lekluppel@hotmail.com
Aline Sebastiani
ORCID: https://orcid.org/0000-0002-2904-5056
Universidade Federal do Paraná, Brasil
E-mail: sebastiani.aline@gmail.com
Rafaela Scariot
ORCID: https://orcid.org/0000-0002-4911-6413
Universidade Federal do Paraná, Brasil
E-mail: rafaela_scariot@yahoo.com.br

\begin{abstract}
Resumo
O objetivo deste estudo foi avaliar o volume de vias aéreas de pacientes com má oclusão esquelética Classe III, submetidos à cirurgia ortognática bimaxilar. A amostra foi composta por 10 pacientes com má oclusão esquelética Classe III, submetidos à cirurgia ortognática bimaxilar. Através da tomografia computadorizada, as medidas volumétricas tridimensionais das vias aéreas foram determinadas utilizando o programa Dolphin Imaging® 11.7 e foram comparadas entre os períodos pré e pós-operatório. Os pacientes foram divididos em dois grupos, (1) diminuição das vias aéreas e (2) aumento das vias aéreas. O grupo 1 apresentou uma redução média de 66,16 $\pm 52,48$ $\mathrm{mm}^{2}$ na avaliação bidimensional das vias aéreas e $2311,16 \pm 1653,71 \mathrm{~mm}^{3}$ na avaliação tridimensional $(\mathrm{p} \leq 0,05)$. Por outro lado, o grupo 2 aumentou 166,5 $\pm 73,13 \mathrm{~mm}^{2}$ e $4116 \pm 1323,85 \mathrm{~mm}^{3}$. Não houve correlação entre os valores obtidos com o movimento ósseo, em $\mathrm{mm}(\mathrm{p} \geq 0,05)$. Pacientes com deformidade classe III submetidos a cirurgia bimaxilar, a maioria com diminuição das vias aéreas, no entanto, essa diminuição nem sempre ocorre na mesma magnitude para todos os pacientes.
\end{abstract}

Palavras-chave: Cirurgia ortognática; Remodelação das vias aéreas; Orofaringe; Nasofaringe; Tomografia.

\begin{abstract}
The aim of this study was to assess the airway volume of patients with Class III skeletal malocclusion undergoing bimaxillary orthognathic surgery. The sample consisted of 10 patients with Class III skeletal malocclusion undergoing bimaxillary orthognathic surgery. Through computed tomography, the three-dimensional volumetric measurements of the airways were determined using the Dolphin Imaging ${ }^{\circledR} 11.7$ program and were compared between the pre- and postoperative periods. Patients were divided into two groups, (1) airway narrowing and (2) airway enlargement. Group 1 showed a mean reduction of $66.16 \pm 52.48 \mathrm{~mm}^{2}$ in the two-dimensional assessment of the airways and $2311.16 \pm 1653.71 \mathrm{~mm}^{3}$ in the three-dimensional assessment $(\mathrm{p} \leq 0.05)$. On the other hand, group 2 increased $166.5 \pm$ $73.13 \mathrm{~mm}^{2}$ and $4116 \pm 1323.85 \mathrm{~mm}^{3}$. There was no correlation between the values obtained with bone movement, in $\mathrm{mm}(\mathrm{p} \geq 0.05)$. Patients with class III deformities undergoing bimaxillary surgery, most with reduced airways, however, this decrease does not always occur to the same extent for all patients.
\end{abstract}

Keywords: Orthognathic surgery; Airway remodeling; Oropharynx; Nasopharynx; Tomography. 


\section{Resumen}

El objetivo de este estudio fue evaluar el volumen de la vía aérea de pacientes con maloclusión esquelética Clase III sometidos a cirugía ortognática bimaxilar. La muestra estuvo constituida por 10 pacientes con maloclusión esquelética clase III sometidos a cirugía ortognática bimaxilar. Mediante tomografía computarizada se determinaron las medidas volumétricas tridimensionales de las vías respiratorias mediante el programa Dolphin Imaging ${ }^{\circledR} 11.7$ y se compararon entre los periodos pre y posoperatorio. Los pacientes se dividieron en dos grupos, (1) estrechamiento de las vías respiratorias y (2) agrandamiento de las vías respiratorias. El grupo 1 mostró una reducción media de 66,16 $\pm 52,48$ $\mathrm{mm}^{2}$ en la evaluación bidimensional de las vías respiratorias y $2311,16 \pm 1653,71 \mathrm{~mm}^{3}$ en la evaluación tridimensional $(\mathrm{p} \leq 0,05)$. Por otro lado, el grupo 2 aumentó $166,5 \pm 73,13 \mathrm{~mm}^{2}$ y $4116 \pm 1323,85 \mathrm{~mm}^{3}$. No hubo correlación entre los valores obtenidos con el movimiento óseo, en $\mathrm{mm}$ ( $\mathrm{p} \geq 0,05)$. Pacientes con deformidades de clase III sometidos a cirugía bimaxilar, la mayoría con vías respiratorias reducidas, sin embargo, esta disminución no siempre ocurre en la misma medida en todos los pacientes.

Palabras clave: Cirugía ortognática; Remodelación de las vías aéreas; Orofaringe; Nasofaringe; Tomografía.

\section{Introdução}

A deformidade dentofacial (DDF) é definida como desproporções dentárias e faciais decorrentes de alterações de crescimento dos ossos face, principalmente da maxila e da mandíbula (Proffit; White; Sarver., 2003). A DDF é evidenciada durante o crescimento e desenvolvimento do indivíduo, criando problemas funcionais, degenerativos, estéticos e psicossociais (American Association of Oral And Maxillofacial Surgeons; 2012). Ela também pode acometer uma ou duas bases ósseas, nos planos vertical, horizontal e transversal, tanto de maneira isolada como combinada, sendo majoritariamente dependentes de componentes ambientais e genéticos (van Spronsen, 2010).

A má oclusão esquelética de classe III pode ser causada devido a um prognatismo mandibular, mas a deficiência de crescimento anteroposterior da maxila é considerada como o principal fator etiológico desta má oclusão. As duas condições ainda podem estar associadas (Oltramari-Navarro et al., 2013). A cirurgia ortognática, para correção destas deformidades, resulta na movimentação das bases ósseas, gerando modificações nos tecidos moles que promovem alterações da via aérea superior devido ao reposicionamento do osso hióide, língua e palato mole (Foronda \& Melhem Elias, 2011; Mattos et al., 2011).

A via aérea superior (Figura 1) consiste em um tubo (de 12 a $15 \mathrm{~cm}$ de comprimento) que se estende desde as narinas até a laringe, ou seja, até o nível da sexta vértebra cervical e é dividida em nasofaringe, orofaringe e laringofaringe (Sheng et al., 2009; Sologuren, 2009). A nasofaringe, está localizada entre os cornetos nasais e o palato duro; a orofaringe, do palato duro até o ápice da epiglote, podendo ser subdividida em orofaringe retropalatina e retroglossal; e a laringofaringe, da base da epiglote até a laringe (Ayappa \& Rapoport, 2003; Sheng et al., 2009). A parede da via aérea é constituída apenas por tecido mole, não possuindo, portanto, suporte por estrutura rígida, como osso ou cartilagem, dessa forma, o suporte mecânico que garante a manutenção da abertura dessa estrutura frente à pressão negativa durante os movimentos de inspiração resulta da tensão e contração da musculatura ao seu redor (Rajagopal \& Paul, 2005). Além da anatomia do tecido mole e esquelético, o espaço aéreo depende de algumas variáveis dinâmicas como volume pulmonar, pressão intraluminal e extraluminal, tonicidade muscular e posição de cabeça (Hinton et al., 1987). 
Figura 1. Segmentos da faringe.

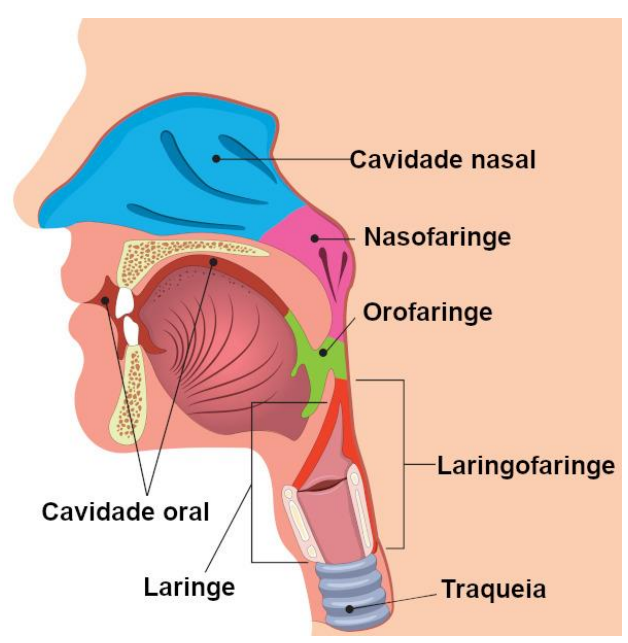

Fonte: Biologianet (2022).

A tomografia computadorizada de feixe cônico (TCFC) tem se tornado uma ferramenta importante para o estudo das vias aéreas. Após a reformatação e exportação dos dados brutos da TCFC, por meio de softwares de processamento de imagem que permitem a sintetização de várias imagens multiplanares (axiais, coronais e sagitais), é possível visualizar a reconstrução tridimensional dos tecidos mineralizados e dos espaços aéreos da região maxilofacial com mínima distorção. Desta forma, torna-se possível a quantificação precisa da morfologia das vias aéreas antes e após os tratamentos cirúrgicos dos pacientes com deformidade dentofacial (Aboudara et al., 2009; de Souza Carvalho et al., 2012).

Este trabalho tem por objetivo demonstrar uma série de casos com as alterações do volume das vias aéreas dos pacientes com má oclusão esquelética de classe III que se submeteram a cirurgia ortognática bimaxilar, através de tomografia computadorizada cone-beam pré-operatória e pós-operatória, utilizando o programa Dolphin Imaging ${ }^{\circledR}$ 11.7.

\section{Metodologia}

\subsection{Comitê de Ética e Pesquisa}

Este estudo foi aprovado pelo Comitê de Ética e Pesquisa da Universidade Positivo, Curitiba, Paraná, Brasil, a partir do número do protocolo: 90735618.5.0000.0093 e seguiu as diretrizes da declaração de Helsinque. Os pacientes assinaram um termo de consentimento livre e esclarecido.

\subsection{Amostra}

Foram selecionadas pacientes com má oclusão esquelética de Classe III submetidos a tratamento ortodônticocirúrgico no Departamento de Cirurgia Oral e Maxilofacial da Universidade Positivo entre fevereiro a julho de 2019. Os critérios de inclusão foram pacientes com má oclusão esquelética de Classe III, submetidos a cirurgia ortognática bimaxilar, que realizaram tomografia computadorizada pré e pós-operatória. Os critérios de exclusão foram cirurgias com manipulação do plano oclusal e ausência da tomografia nos tempos analisados. Dados como sexo, idade, movimento cirúrgico realizado e quantificação do movimento realizado também foram catalogados.

\subsection{Avaliação Tomográfica}

Foram realizadas duas tomografias para cada paciente, uma no pré-operatório, após a finalização do preparo ortocirúrgico e uma no pós-operatório de pelo menos seis meses após o procedimento. As imagens foram adquiridas no dispositivo 
de imagem Scanner Classic iCAT, Imaging Sciences International, seguindo o mesmo protocolo: tempo de aquisição da imagem de 20 a 40 segundos com o paciente sentado, usando um colete de proteção, em posição natural da cabeça, respirando calmamente, não deglutindo durante o exame e permanecendo na máxima intercuspidação habitual. O exame foi realizado pelo mesmo operador em uma clínica de radiologia privada.

A partir do exame, os dados foram convertidos para o formato DICOM (Digital Imaging and Communications in Medicine) para posterior análise no programa Dolphin Imaging ${ }^{\circledR} 11.7$ (Dolphin Imaging and Management Solutions, Patterson Technology, Chatsworth, CA, EUA).

\subsection{Delimitação do volume da via aérea}

Para avaliar o espaço aéreo foram usados pontos semelhantes aos descritos por Raffaini \& Pisani, (2013) e Schendel et al., (2014) delimitando a via aérea em limite superior e inferior:

- $\quad$ limite superior (região retropalatal) - linha entre a espinha nasal posterior e a primeira vértebra cervical (Figura 2);

- $\quad$ limite inferior (área retroglossal) - linha entre o osso hióide e a terceira vértebra cervical (Figura 3).

Figura 2. Limite superior (região retropalatal), delimitada por uma linha reta entre a espinha nasal posterior e a primeira vértebra cervical do indivíduo.

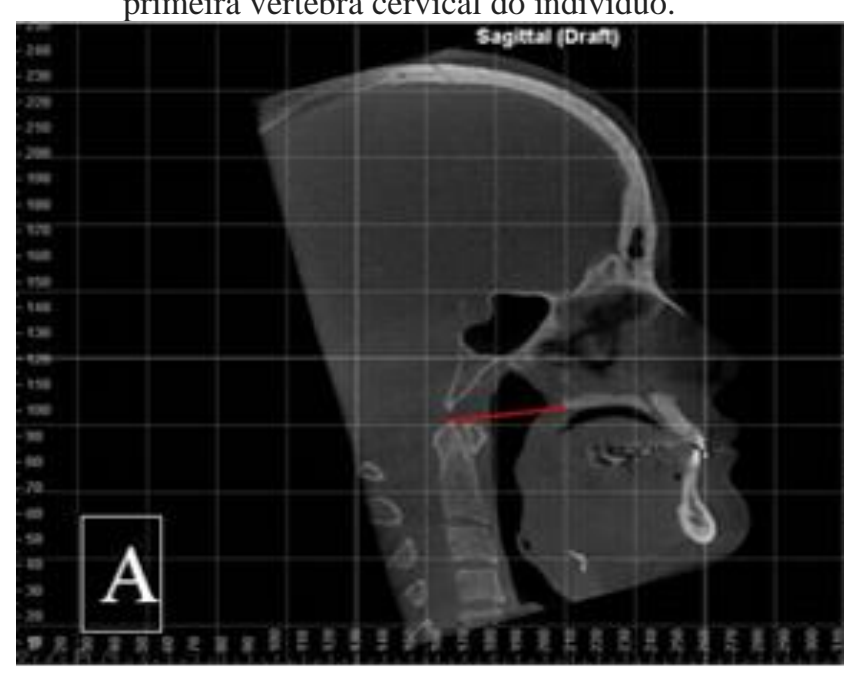

Fonte: Autores. 
Research, Society and Development, v. 11, n. 1, e46711125238, 2022

(CC BY 4.0) | ISSN 2525-3409 | DOI: http://dx.doi.org/10.33448/rsd-v11i1.25238

Figura 3. Limite inferior (área retroglossal), delimitada por uma linha reta entre o osso hióide e a terceira vértebra cervical do indivíduo.



Fonte: Autores.

Com o espaço aéreo delimitado, foram obtidas as medidas de área das vias aéreas em $\mathrm{mm}^{2}$ (Figura 4) e volume em $\mathrm{mm}^{3}$ (Figura 5) dos pacientes no pré e pós-operatório da amostra. Todas as aferições foram realizadas por um profissional (CL), treinado e calibrado.

Figura 4. Reconstrução bidimensional $\left(\mathrm{mm}^{2}\right)$ da área da via aérea a partir dos limites superior e inferior.

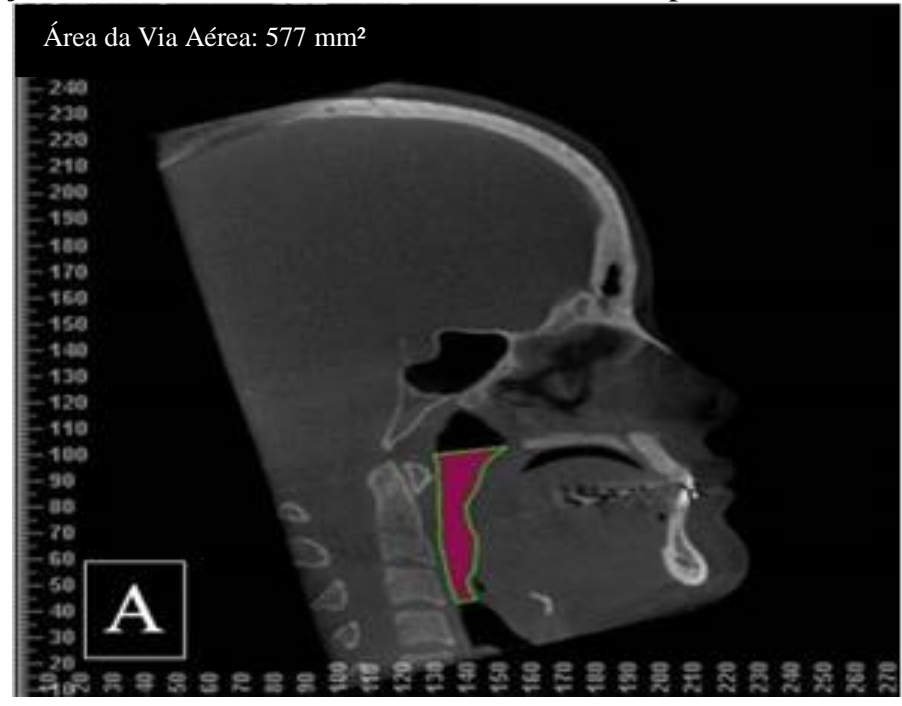

Fonte: Autores. 
Figura 5. Reconstrução tridimensional $\left(\mathrm{mm}^{3}\right)$ da área da via aérea a partir dos limites superior e inferior.

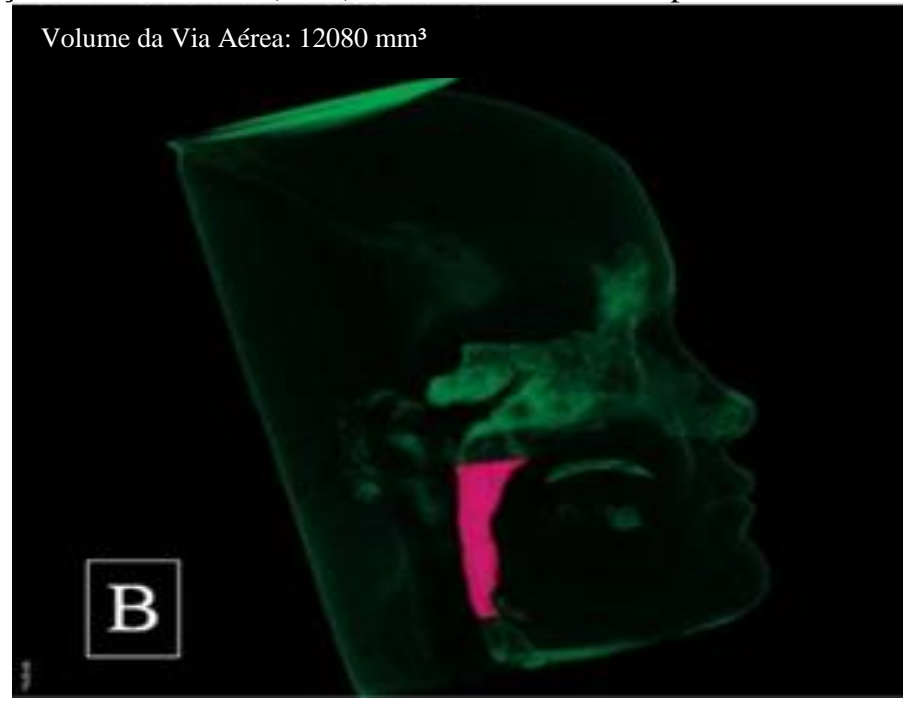

Fonte: Autores.

\subsection{Avaliação das Movimentações}

Também foram analisados os movimentos cirúrgicos através da tomografia computadorizada e do programa Dolphin Imaging®. Foi realizada sobreposição do crânio composto da tomografia pré-operatória com a tomografia do pósoperatório. Foram usados o Ponto A para avaliar a movimentação da maxila e Ponto B para a mandíbula.

\subsection{Análise Estatística}

A análise estatística foi realizada no software SPSS (Statistical Package for the Social Sciences, V.20, IBM, EUA), com nível de significância de 0,05. Para avaliar a normalidade das variáveis, foi utilizado o teste de Shapiro-Wilk. Os dados obtidos foram paramétricos. A análise descritiva foi utilizada para apresentar volumes médios, e desvio padrão. Para a comparação entre a avaliação pré e pós-operatória bidimensional e tridimensional dos mesmos pacientes, foi utilizado o teste $\mathrm{T}$ pareado. O coeficiente de correlação de Pearson foi utilizado para avaliar a correlação entre a quantidade em milímetros de movimento e o volume bidimensional e tridimensional.

\section{Resultados}

Um total de 10 pacientes adultos foram incluídos no estudo, destes, oito eram mulheres. A média de idade foi de $30,4 \operatorname{anos}( \pm 10,8)$.

Dos 10 pacientes submetidos à cirurgia ortognática, em todos os casos a maxila foi avançada, em 9 pacientes a mandíbula foi recuada e em 1 paciente a mandíbula foi avançada. O tempo médio de avaliação tomográfica foi de 9 meses após a cirurgia.

O valor médio da área da via aérea superior calculada pela análise bidimensional foi de $586,6( \pm 135,21) \mathrm{cm}^{2}$ no préoperatório e $613,50( \pm 201,85) \mathrm{cm}^{2}$ no pós-operatório. Já a média de volume das vias aéreas superiores foi $13242,8( \pm 4038,16)$ $\mathrm{cm}^{3}$ no pré-operatório e $13502,50( \pm 5893,78) \mathrm{cm}^{3}$ no pós-operatório.

A tabela 1 demonstra as características da amostra, com as movimentações cirúrgicas realizadas, as medidas de volume da via aérea nos períodos pré e pós-operatórios, bem como a diferença (delta) da área e volume entre os períodos. Nessa tabela podemos observar que a maioria dos pacientes foi submetida a um avanço maxilar associado a um recuo 
mandibular, exceto um paciente que teve a maxila e a mandíbula avançados. As medidas de área e volume da via aérea diminuíram para 6 pacientes no período pós-operatório e aumentaram para 4 pacientes.

Tabela 1. Análise descritiva das características da amostra, movimentações cirúrgicas e dos volumes de via aérea pré e pósoperatórios.

\begin{tabular}{|c|c|c|c|c|c|c|c|c|c|c|c|}
\hline Paciente & Sexo & Idade & $\begin{array}{c}\text { Avanço de } \\
\text { Maxila (mm) }\end{array}$ & $\begin{array}{c}\text { Recuo de } \\
\text { Mandíbula } \\
(\mathbf{m m})\end{array}$ & $\begin{array}{c}\text { Avanço de } \\
\text { Mandíbula } \\
\text { (mm) }\end{array}$ & $\begin{array}{l}\text { Volume } \\
\text { Pré }\left(\mathrm{cm}^{2}\right)\end{array}$ & $\begin{array}{c}\text { Volume Pós } \\
\quad\left(\mathrm{cm}^{2}\right)\end{array}$ & $\begin{array}{l}\text { Delta } \\
\left(\mathbf{c m}^{2}\right)\end{array}$ & $\begin{array}{c}\text { Volume } \\
\text { Pré }\left(\mathbf{c m}^{3}\right)\end{array}$ & $\begin{array}{c}\text { Volume } \\
\text { Pós }\left(\mathrm{cm}^{3}\right)\end{array}$ & $\begin{array}{l}\text { Delta } \\
\left(\mathbf{c m}^{3}\right)\end{array}$ \\
\hline 1 & $\mathrm{~F}$ & 34 & 1.0 & 3.5 & 0 & 621 & 465 & -156 & 15141 & 10984 & -4157 \\
\hline 2 & $\mathrm{~F}$ & 50 & 2.7 & 2.7 & 0 & 609 & 701 & 92 & 15802 & 20019 & 4217 \\
\hline 3 & F & 35 & 3.3 & 3.4 & 0 & 588 & 577 & -11 & 12571 & 12080 & -491 \\
\hline 4 & $\mathrm{~F}$ & 41 & 3.1 & 1.0 & 0 & 560 & 533 & -27 & 9214 & 7804 & -1410 \\
\hline 5 & M & 18 & 3.2 & 2.3 & 0 & 601 & 506 & -95 & 14559 & 10173 & -4386 \\
\hline 6 & $\mathrm{~F}$ & 23 & 1.0 & 3.4 & 0 & 446 & 392 & -54 & 9541 & 8577 & -964 \\
\hline 7 & $\mathrm{~F}$ & 37 & 3.6 & 4.5 & 0 & 443 & 561 & 118 & 8040 & 10263 & 2223 \\
\hline 8 & $\mathrm{~F}$ & 28 & -2.0 & 2.8 & 0 & 431 & 642 & 211 & 11386 & 16569 & 5183 \\
\hline 9 & M & 19 & 4.6 & 0 & 4.3 & 889 & 1128 & 245 & 21764 & 26605 & 4841 \\
\hline 10 & $\mathrm{~F}$ & 19 & 3.0 & 7.0 & 0 & 684 & 630 & -54 & 14410 & 11951 & -2459 \\
\hline
\end{tabular}

Fonte: Autores.

Assim, os pacientes foram divididos em dois grupos de acordo com a diminuição (grupo 1) ou o aumento do espaço da via aérea (grupo 2). A tabela 2 demonstra a comparação das medidas pré e pós-operatório nos dois grupos. O grupo 1 apresentou uma diminuição significativa de $66,16 \pm 52,48 \mathrm{~mm}^{2}$ na área da via aérea avaliada de forma bidimensional $(\mathrm{p}=$ $0,002)$ e $2311,16 \pm 1653,71 \mathrm{~mm}^{3}$ no volume aferido na análise tridimensional $(\mathrm{p}=0,019)$. O grupo 2 também demonstrou aumento significativo de 166,5 $\pm 73,13 \mathrm{~mm}^{2}$ na análise bidimensional $(\mathrm{p}=0,020)$ e $4116 \pm 1323,85 \mathrm{~mm}^{3}$ na análise tridimensional $(\mathrm{p}=0,008)$. 
Tabela 2. Comparação das medidas do espaço da via aérea entre os períodos pré e pós-operatório no grupo com diminuição e no grupo com aumento do espaço das vias aéreas superiores.

\begin{tabular}{|c|c|c|c|c|c|}
\hline & & $\begin{array}{l}\text { Pré-operatório } \\
\text { Média } \pm \text { DP }\end{array}$ & $\begin{array}{l}\text { Pós-operatório } \\
\text { Média } \pm \text { DP }\end{array}$ & $\begin{array}{c}\text { DELTA } \\
\text { Média } \pm \text { DP }\end{array}$ & Valor-P \\
\hline \multirow[t]{2}{*}{ Redução de vias aéreas } & Análise bidimensional $\left(\mathrm{mm}^{2}\right)$ & $\begin{array}{l}583.33 \\
\pm 79.10\end{array}$ & $\begin{array}{l}517.16 \\
\pm 83.75\end{array}$ & $\begin{array}{l}-66.16 \\
\pm 52.48\end{array}$ & 0.002 \\
\hline & Análise tridimensional $\left(\mathrm{mm}^{2}\right)$ & $\begin{array}{r}12572.66 \\
\pm 2622.56\end{array}$ & $\begin{array}{r}10261.50 \\
\pm 1764.54\end{array}$ & $\begin{array}{l}-2311.16 \\
\pm 1653.71\end{array}$ & 0.019 \\
\hline \multirow[t]{2}{*}{ Aumento de vias aéreas } & Análise bidimensional $\left(\mathrm{mm}^{2}\right)$ & $\begin{array}{r}591.50 \\
\pm 210.62\end{array}$ & $\begin{array}{r}758.00 \\
\pm 253.25\end{array}$ & $\begin{array}{l}+166.50 \\
\pm 73.13\end{array}$ & 0.020 \\
\hline & Análise tridimensional $\left(\mathrm{mm}^{2}\right)$ & $\begin{array}{r}14248.00 \\
\pm 5933.95\end{array}$ & $\begin{array}{r}18364.00 \\
\pm 6819.12\end{array}$ & $\begin{array}{l}+4116.00 \\
\pm 1323.85\end{array}$ & 0.008 \\
\hline
\end{tabular}

Nota: Teste-t de Student pareado, com nível de significância 0.05. Fonte: Autores.

A comparação das características dos pacientes e das movimentações cirúrgicas entre os dois grupos pode ser visualizadas na tabela 3. No grupo 1, 5 pacientes eram do gênero feminino e 1 masculino. Já no grupo 2, 3 pacientes eram do gênero feminino e 1 masculino. A média de idade no grupo 1 foi de $28 \pm 9,58$ anos, enquanto no grupo 2 a média de idade foi $33 \pm 13$ anos. O avanço da maxila no grupo 1 foi de $2,43 \pm 1,11 \mathrm{~mm}$ e no grupo 2 foi de $2,77 \pm 18,83 \mathrm{~mm}$. O recuo da mandíbula no grupo 1 foi de 3,43 $\pm 1,99 \mathrm{~mm}$ e o recuo da mandíbula no grupo 2 foi de 3,57 $\pm 9,57 \mathrm{~mm}$.

Tabela 3. Avaliação descritiva das variáveis do paciente e da cirurgia nos grupos com diminuição e aumento do espaço da via aérea superior

\begin{tabular}{|c|c|c|c|c|}
\hline & & & Vias Aéreas Diminuídas & Vias Aéreas Aumentadas \\
\hline \multirow[t]{2}{*}{ Sexo } & Feminino & $\mathrm{N}(\%)$ & $5(83.3)$ & $3(75)$ \\
\hline & Masculino & $\mathrm{N}(\%)$ & $1(16.7)$ & $1(25)$ \\
\hline \multicolumn{2}{|c|}{ Idade (anos) } & Média + D.P. & $28.33(9.58)$ & $33.5(13.22)$ \\
\hline \multicolumn{2}{|c|}{ Avanço de Maxila } & $\mathrm{N}(\%)$ & $6(100)$ & $4(100)$ \\
\hline \multicolumn{2}{|c|}{ Recuo de Mandíbula } & $\mathrm{N}(\%)$ & $6(100)$ & $3(75)$ \\
\hline \multicolumn{2}{|c|}{ Avanço de Mandíbula } & $\mathrm{N}(\%)$ & $0(0)$ & $1(25)$ \\
\hline \multicolumn{2}{|c|}{ Avanço de Maxila (mm) } & Média + D.P. & $2.43 \pm 1.11$ & $2.77 \pm 18.83$ \\
\hline \multicolumn{2}{|c|}{ Recuo de Mandíbula (mm) } & Média + D.P. & $3.43 \pm 1.99$ & $3.57 \quad 9.57$ \\
\hline
\end{tabular}

Fonte: Autores.

\section{Discussão}

Diversos estudos têm demonstrado o efeito da cirurgia ortognática nas vias aéreas superiores. Isso ocorre porque a maxila e a mandíbula são as bases dos tecidos moles da região orofaríngea, assim, seu posicionamento tem importante influência na estabilização destes tecidos. Tal relação é tão importante que a cirurgia ortognática de avanço bimaxilar é um 
dos tratamentos cirúrgicos mais efetivos para a apnéia obstrutiva do sono (Alcalde et al., 2019; Daluz et al., 2021; Yang et al., 2020).

Nesse contexto, os softwares tridimensionais (3D) revolucionaram o planejamento em cirurgia ortognática. Esta tecnologia permite a simulação de movimentos cirúrgicos e avaliação do volume e área do espaço aéreo faríngeo, que não é possível com o software bidimensional (2D) (Azambuja; et al., 2019). A avaliação tridimensional oferece uma série de vantagens: realizam as medições do volume com maior precisão, melhor localização dos pontos de referência, reprodução e reconstrução de imagens 3D, sem perder a qualidade (Li, et al., 2014). O software Dolphin Imaging® 3D oferece uma previsão precisa das vias aéreas que é uma etapa importante no planejamento virtual em 3D (Elshebiny et al., 2020).

Um possível viés dos estudos que avaliam volume de vias aéreas é o posicionamento inadequado do paciente durante a realização da tomografia. A postura e posição da cabeça devem ser realizadas de forma adequada e padronizada, pois a região orofaríngea diminui com uma mudança da posição vertical para a posição supina. Essa mudança na postura corporal, afeta o tamanho das vias aéreas superiores devido aos efeitos da musculatura periférica (Kawakami, et al., 2005). Muto et al., (2002) mostraram que um aumento de 10 graus na inclinação craniocervical aumentou o espaço aéreo posterior em aproximadamente $4 \mathrm{~mm}$. Assim, protocolos para garantir a adequada posição da cabeça durante a realização da tomografia, mantendo o mesmo exato posicionamento no exame pré e pós-operatório é fundamental. Em nosso estudo fizemos a padronização das condições do paciente e o exame foi realizado sempre pelo mesmo operador.

As mais diversas movimentações cirúrgicas têm sido estudadas por seus efeitos nas vias aéreas, pois ao mesmo tempo em que o avanço das bases ósseas pode produzir um aumento do espaço da via aérea, o recuo pode causar uma diminuição (He et al., 2019). Essa preocupação com as vias aéreas vem ganhando importância há muitos anos, pois sabe-se que as alterações respiratórias podem levar a diversas alterações de saúde e diminuir a expectativa de vida do paciente (Daluz et al., 2021; L. He et al., 2019). No passado, os pacientes Classe III eram tratados em sua maioria por recuos mandibulares, isso ocorria devido a falhas no diagnóstico e devido a dificuldades técnicas para operar a maxila (J. He et al., 2017; Yang et al., 2020). Atualmente, fica evidenciado a preferência dos cirurgiões em avançar a maxila ao invés de recuar a mandíbula, e na maioria dos casos, a principal alteração de crescimento está presente na maxila (Daluz et al., 2021; Yang et al., 2020). Muitos pacientes são submetidos a cirurgia combinada de avanço maxilar e recuo mandibular devido a severidade da má-oclusão e ao envolvimento de ambas as bases ósseas na deformidade.

Estudos tem relatado não haver uma diminuição significativa do volume da via aérea em cirurgias combinadas (He et al., 2016). Apesar de não serem diferenças significativas, alguns estudos demonstraram uma diferença média positiva (Azevêdo et al., 2016; Burkhard et al., 2014; Gokce et al., 2014 ref) e outros estudos apresentam uma diferença média negativa (Hatab et al., 2015; Kim et al., 2016; Hsieh et al., 2016) ao comparar os períodos pré e pós-operatórios. Provavelmente estes estudos avaliaram em conjunto pacientes que tiveram diminuição da via aérea com pacientes que tiveram aumento.

O estudo de Tan et al., (2017) analisou revisões sistemáticas e a partir de seus resultados obteve a conclusão de que há evidencias que a cirurgia ortognática bimaxilar deve ser considerada como o método de escolha, principalmente nos casos graves de má oclusão esquelética classe III. A cirurgia ortognática bimaxilar promove uma menor diminuição nas vias aéreas superiores comparada a cirurgia de recuo mandibular isolada para correção de má oclusão esquelética em pacientes classe III (He et al., 2017). Já foi demonstrada: recuos mandibulares isolados em pacientes classe III contraem as vias aéreas da orofaringe e hipofaringe, devido a alterações na posição da língua e do osso hióide (Foronda \& Melhem Elias, 2011). Entretanto, com o avanço simultâneo da maxila, limita a constrição da via aérea total e causa aumento do volume nasofaríngeo (MCINTYRE, 2011). 
De acordo com Carvalho et al., (2012), embora pareça que o avanço da maxila resulte em aumento do volume das vias aéreas em alguns pacientes, não é possível determinar a estabilidade a longo prazo desse ganho pois o benefício cirúrgico pode ser perdido com o envelhecimento, principalmente devido à redução da elasticidade e ganho de peso (De Souza Carvalho, et al., 2012). De acordo com Riley et al., (1987) ganhos no volume da via aérea superior se tornam relevantes somente quando a maxila é avançada entre 8 e $12 \mathrm{~mm}$, o que não é um movimento usual na rotina das cirurgias ortognáticas.

Quando os movimentos de avanço maxilar e recuo mandibular são combinados, torna-se menor a previsibilidade quanto aos efeitos sobre as vias aéreas. Embora o recuo da mandíbula promova redução do volume em certas regiões, o avanço da maxila pode compensá-lo promovendo o aumento em outros locais. Com isso, o real efeito dependerá diretamente da proporção de ganho e perda de volume resultantes (Giralt-Hernando et al., 2021; J. He et al., 2017; Yang et al., 2020).

Em nosso estudo, encontramos que parte dos pacientes teve um aumento significativo das vias aéreas e outro grupo apresentou uma diminuição significativa. El \& Palomo (2013) observaram que a adaptação muscular ocorre durante os primeiros 6 meses após a cirurgia e avaliações realizadas antes desse período podem limitar a capacidade de precisão dos valores encontrados. Com isso, nossa avaliação foi conduzida com seis meses de pós-operatório. Entretanto não foi possível identificar as variáveis preditoras para estes diferentes resultados por se tratar de um estudo com um pequeno número amostral. É de fundamental importância compreender melhor as alterações do volume de via aérea após esta modalidade de cirurgia e quais outras variáveis podem influenciar. Muitos pacientes que buscam corrigir sua oclusão e estética facial para a melhora da qualidade de vida podem acabar afetando negativamente a respiração. Além disso, muitos pacientes que buscam a cirurgia já apresentam histórico de ronco e outras alterações respiratórias, devendo ser adequadamente informados dos riscos e benefícios da cirurgia em relação a isso.

Nosso estudo sinaliza para a possibilidade de aumentos e diminuição significativa das vias aéreas após cirurgia bimaxilar para a correção de má-oclusão esquelética de Classe III. Entretanto, a principal limitação do nosso estudo foi o pequeno número de pacientes, dificultando a identificação de outros fatores individuais dos pacientes e das movimentações cirúrgicas que possam predizer o aumento ou a diminuição do volume das vias aéreas. Além disso, em nosso estudo avaliamos a alteração total do volume da faringe, futuros estudos com um número maior de pacientes, devem investigar as diferenças de volume em áreas específicas como a nasofaringe e a área transversal mínima. E além da análise tomográfica é necessário a análise de outros parâmetros para poder afirmar que isso tem uma real repercussão clínica. Novos estudos avaliando também exames dos tecidos moles como a videonasofaringoscopia, endoscopia do sono, questionários de sono, polissonografias devem ser realizados.

\section{Conclusão}

Assim, concluímos que a cirurgia ortognática combinada em pacientes com deformidade dentofacial de classe III influencia na via aérea superior, podendo aumentar ou diminuir significativamente seu volume.

Estudos adicionais são necessários para definir a estabilidade das mudanças volumétricas em longo prazo e a sua repercussão clínica nos pacientes, bem como identificar preditores cirúrgicos e individuais para o aumento ou a diminuição da via aérea superior após o tratamento cirúrgico. Estudos futuros devem ser feitos com um número maior de pacientes, envolver mais áreas da via aérea para medições e testar outros métodos de avaliação para comparação e obtenção de resultados mais próximo ao real possível. 


\section{Referências}

Aboudara, C., Nielsen, I., Huang, J. C., Maki, K., Miller, A. J., \& Hatcher, D. (2009). Comparison of airway space with conventional lateral headfilms and 3 dimensional reconstruction from cone-beam computed tomography. American Journal of Orthodontics and Dentofacial Orthopedics, 135(4), 468-479. https://doi.org/10.1016/j.ajodo.2007.04.043

Alcalde, L. F. A., Faria, P. E. P., Nogueira, R. L. M., Chihara, L., \& Sant'Ana, E. (2019a). Computed tomography visualizing alterations in the upper airway after orthognathic surgery. Journal of Cranio-Maxillofacial Surgery, 47(7), 1041-1045. https://doi.org/10.1016/j.jcms.2019.04.006

American association of oral and maxillofacial surgeons. Parameters of care: clinical practice guidelines for oral and maxillofacial surgery (AAOMS ParCare2012). J Oral Maxillofac Surg. 2012;70(11):107-36.

Ayappa, I., \& Rapoport, D. M. (2003). The upper airway in sleep: Physiology of the pharynx. In Sleep Medicine Reviews (Vol. 7, Issue 1, pp. 9-33). W.B. Saunders Ltd. https://doi.org/10.1053/smrv.2002.0238

Azevêdo, M. S., Machado, A. W., da Silva Barbosa, I., Esteves, L. S., Rocha, V. Á. C., \& Bittencourt, M. A. V. (2016). Evaluation of upper airways after bimaxillary orthognathic surgery in patients with skeletal class III pattern using cone-beam computed tomography. Dental Press Journal of Orthodontics, 21(1), 34-41. https://doi.org/10.1590/2177-6709.21.1.034-041.oar

Azambuja Alcalde, L. F., Pinto Faria, P. E., Maia Nogueira, R. L., Chihara, L., \& Sant'Ana, E. (2019). Computed tomography visualizing alterations in the upper airway after orthognathic surgery. Journal of Cranio-Maxillofacial Surgery. doi: 10.1016/j.jcms.2019.04.006.

Burkhard, J. P. M., Dietrich, A. D., Jacobsen, C., Roos, M., Lübbers, H. T., \& Obwegeser, J. A. (2014). Cephalometric and three-dimensional assessment of the posterior airway space and imaging software reliability analysis before and after orthognathic surgery. Journal of Cranio-Maxillofacial Surgery, 42(7), 1428-1436. https://doi.org/10.1016/j.jcms.2014.04.005

Daluz, A. D. J., da Silva, T. V. S., Tôrres, B. O., Costa, D. F. N., \& Santos, L. A. de M. (2021). Long-term airway evolution after orthognathic surgery: Systematic Review. In Journal of Stomatology, Oral and Maxillofacial Surgery. Elsevier Masson s.r.1. https://doi.org/10.1016/j.jormas.2021.04.006

de Souza Carvalho, A. C. G., Magro Filho, O., Garcia, I. R., Araujo, P. M., \& Nogueira, R. L. M. (2012). Cephalometric and three-dimensional assessment of superior posterior airway space after maxillomandibular advancement. International Journal of Oral and Maxillofacial Surgery, 41(9), 1102-1111. https://doi.org/10.1016/j.ijom.2012.05.009

Elshebiny, T., Bous, R., Withana, T., Morcos, S., \& Valiathan, M. (2020). Accuracy of Three-Dimensional Upper Airway Prediction in Orthognathic Patients Using Dolphin Three-Dimensional Software. The Journal of Craniofacial Surgery, 31(4), 1098-1100. https://doi.org/10.1097/SCS.0000000000006566

Foronda, R., \& Melhem Elias, F. (2011). Avaliação de dois programas de computador na previsão do perfil facial de pacientes submetidos à cirurgia ortognática (Vol. 18, Issue 4).

Giralt-Hernando, M., Valls-Ontañón, A., Haas Junior, O. L., Masià-Gridilla, J., \& Hernández-Alfaro, F. (2021). What are the Surgical Movements in Orthognathic Surgery That Most Affect the Upper Airways? A Three-Dimensional Analysis. Journal of Oral and Maxillofacial Surgery, 79(2), 450-462. https://doi.org/10.1016/j.joms.2020.10.017

Gokce, S. M., Gorgulu, S., Gokce, H. S., Bengi, A. O., Karacayli, U., \& Ors, F. (2014). Evaluation of pharyngeal airway space changes after bimaxillary orthognathic surgery with a 3-dimensional simulation and modeling program. American Journal of Orthodontics and Dentofacial Orthopedics, 146(4), 477492. https://doi.org/10.1016/j.ajodo.2014.06.017

Hatab, N. A., Konstantinović, V. S., \& Mudrak, J. K. H. (2015). Pharyngeal airway changes after mono- and bimaxillary surgery in skeletal class III patients: Cone-beam computed tomography evaluation. Journal of Cranio-Maxillofacial Surgery, 43(4), 491-496. https://doi.org/10.1016/j.jcms.2015.02.007

He, J., Wang, Y., Hu, H., Liao, Q., Zhang, W., Xiang, X., \& Fan, X. (2017). Impact on the upper airway space of different types of orthognathic surgery for the correction of skeletal class III malocclusion: A systematic review and meta-analysis. In International Journal of Surgery (Vol. 38, pp. 31-40). Elsevier Ltd. https://doi.org/10.1016/j.ijsu.2016.12.033

He, L., He, S., Wu, X., \& Huang, Y. (2019). Three-Dimensional Morphological Changes of the Upper Airway in Patients With Skeletal Class III Malocclusion After Orthognathic Surgery. The Journal of Craniofacial Surgery, 30(8), 2451-2455. https://doi.org/10.1097/SCS.0000000000005738

Hsieh Y.J, Chen Y.C., Chen Y.A., Liao Y.F., Chen Y.R. Effect of bimaxillary rotational setback surgery on upper airway structure in skeletal class III deformities. Plast Reconstr Surg. 2015;135(2):361e-9e. doi:10.1097/PRS.0000000000000913

Hinton, V. A., Warren, D. W., Hairfield, W. M., \& Seaton, D. (1987). The relationship between nasal cross-sectional area and nasal air volume in normal and nasally impaired adults. American Journal of Orthodontics and Dentofacial Orthopedics, 92(4), 294-298. https://doi.org/10.1016/0889-5406(87)90329-5

Hsieh, Y. J., Chen, Y. C., Chen, Y. A., Liao, Y. F., \& Chen, Y. R. (2015). Effect of bimaxillary rotational setback surgery on upper airway structure in skeletal class III deformities. Plastic and Reconstructive Surgery, 135(2), 361e-369e. https://doi.org/10.1097/PRS.0000000000000913

Kawakami, M., Yamamoto, K., Fujimoto, M., Ohgi, K., Inoue, M., \& Kirita, T. (2005). Changes in tongue and hyoid positions, and posterior airway space following mandibular setback surgery. Journal of Cranio-Maxillofacial Surgery, 33(2), 107-110. https://doi.org/10.1016/j.jcms.2004.10.005

Kim, H. S., Kim, G. T., Kim, S., Lee, J. W., Kim, E. C., \& Kwon, Y. D. (2016). Three-dimensional evaluation of the pharyngeal airway using cone-beam computed tomography following bimaxillary orthognathic surgery in skeletal class III patients. Clinical Oral Investigations, 20(5), 915-922. https://doi.org/10.1007/s00784-015-1575-4

Li, L., Liu, H., Cheng, H., Han, Y., Wang, C., Chen, Y., Song, J., \& Liu, D. (2014). CBCT Evaluation of the upper airway morphological changes in growing patients of class ii division 1 malocclusion with mandibular retrusion using twin block appliance: A comparative research. PLoS ONE, 9(4). https://doi.org/10.1371/journal.pone.0094378 
Mattos, C. T., Vilani, G. N. L., Sant'Anna, E. F., Ruellas, A. C. O., \& Maia, L. C. (2011). Effects of orthognathic surgery on oropharyngeal airway: A metaanalysis. In International Journal of Oral and Maxillofacial Surgery (Vol. 40, Issue 12, pp. 1347-1356). https://doi.org/10.1016/j.ijom.2011.06.020

McIntyre B.P. Volumetric airway changes in patients undergoing orthognathic surgery: a conebeam CT evaluation [thesis]. Oklahoma City, Okla: University of Oklahoma; 2011.

Muto, T., Takeda, S., Kanazawa, M., Yamazaki, A., Fujiwara, Y., \& Mizoguchi, I. (2002). The effect of head posture on the pharyngeal airway space (PAS). International Journal of Oral and Maxillofacial Surgery, 31(6), 579-583. https://doi.org/10.1054/ijom.2002.0279

Oltramari-Navarro, P. V. P., Almeida, R. R. de, Conti, A. C. de C. F., Navarro, R. de L., Almeida, M. R. de, \& Fernandes, L. S. A. F. P. (2013). Early treatment protocol for skeletal class III malocclusion. Brazilian Dental Journal, 24(2), 167-173. https://doi.org/10.1590/0103-6440201301588

Proffit W.R., White R.P., Sarver D.M. Contemporary Treatment of Dentofacial Deformity. Preface. In: Proffit WR, White RP, Sarver DM, editors. CV Mosby; St Louis, Mo: 2003. p. vii.

Raffaini, M., \& Pisani, C. (2013). Clinical and cone-beam computed tomography evaluation of the three-dimensional increase in pharyngeal airway space following maxillo-mandibular rotation-advancement for Class II-correction in patients without sleep apnoea (OSA). Journal of Cranio-Maxillofacial Surgery, 41(7), 552-557. https://doi.org/10.1016/j.jcms.2012.11.022

Rajagopal, M. R., \& Paul, J. (2005). 251-256 applied anatomy and physiology of the airway and breathing. In Indian J. Anaesth (Vol. 49, Issue 4). http://journals.lww.com/ijaweb

Riley, R. W., Powell, N. B., \& Ware, W. (1997). Obstructive Sleep Apnea Syndrome Folio wing Surgery for Mandibular Prognathism. In J Oral Maxillofac Surg (Vol. 45).

Schendel, S. A., Broujerdi, J. A., \& Jacobson, R. L. (2014). Three-dimensional upper-airway changes with maxillomandibular advancement for obstructive sleep apnea treatment. American Journal of Orthodontics and Dentofacial Orthopedics, 146(3), 385-393. https://doi.org/10.1016/j.ajodo.2014.01.026

Sheng, C. M., Lin, L. H., Su, Y., \& Tsai, H. H. (2009). Developmental changes in pharyngeal airway depth and hyoid bone position from childhood to young adulthood. Angle Orthodontist, 79(3), 484-490. https://doi.org/10.2319/062308-328.1

Sologuren, N. (n.d.). Anatomía de la vía aérea. Retrieved January 4, 2022, from https://revistachilenadeanestesia.cl/anatomia-de-la-via-aerea/

Tan, S. K., Leung, W. K., Tang, A. T. H., \& Zwahlen, R. A. (2017). Letter to the editor on the article "Impact on the upper airway space of different types of orthognathic surgery for the correction of skeletal class III malocclusion: A systematic review and meta-analysis.” In International Journal of Surgery (Vol. 45, pp. 156-157). Elsevier Ltd. https://doi.org/10.1016/j.ijsu.2017.03.083

van Spronsen, P. H. (2010). Long-Face Craniofacial Morphology: Cause or Effect of Weak Masticatory Musculature? Seminars in Orthodontics, 16(2), 99117. https://doi.org/10.1053/j.sodo.2010.02.001

Yang, H. J., Jung, Y. E., Kwon, I. J., Lee, J. Y., \& Hwang, S. J. (2020). Airway changes and prevalence of obstructive sleep apnoea after bimaxillary orthognathic surgery with large mandibular setback. International Journal of Oral and Maxillofacial Surgery, 49(3), 342-349. https://doi.org/10.1016/j.ijom.2019.07.012 